\title{
Diagnosis and Surgical Treatment of Epidural Varicose Veins of the Lumbar Spine
}

\section{Kayode Agboola ${ }^{1 *}$ and Eugene Slynko ${ }^{2}$}

${ }^{1}$ Romodanov Institute of Neurosurgery, National Academy of Medical Sciences of Ukraine, Kyiv, Ukraine

${ }^{2}$ Professor and Head of Department, Department of Spinal Pathologies,

Romodanov, Institute of Neurosurgery, National Academy of Medical Sciences of Ukraine, Kyiv, Ukraine

*Corresponding Author: Kayode Agboola, Romodanov Institute of Neurosurgery, National Academy of Medical Sciences of Ukraine, Kyiv, Ukraine.
Received: July 14, 2020

Published: February 15, 2021

(C) All rights are reserved by Kayode Agboola and Eugene Slynko.

\begin{abstract}
Introduction: Dilated lumbar epidural veins are vascular abnormalities causing compression, ischemic injury and excessive pressure on spinal nerve roots, theca sac or dorsal ganglia, giving rise to symptoms of lumbar radiculopathy of the involved neural structures. Degenerative changes of the spine might also be observed.

Importance: The importance of mentioned pathology is in the complexity of its diagnosis - missed diagnosis, relatively small number of observations, classification types, publications and estimates of the long-term outcomes of surgical treatment of this pathology on follow-ups. Misdiagnosis is often encountered due to low awareness even on MRI.

Objectives: The aim of this study was to increase the awareness and lay emphasis on the importance of diagnosis, classification, pattern of occurrence, and surgical treatment of lumbar epidural varices.

Observation: $\mathrm{N}=100$.

Diagnostic Method: Enhanced lumbar MRI and occasional CT.

Additional Diagnostic Method: Venospondylography.

Surgical Intervention: Laminectomy and microscopic decompression using micro-coagulation - bipolar cautery and eventual excision.

Outcomes: $65.0 \%, 27.0 \%$ and $8.0 \%$ segmentary, local and diffused varices respectively in two cohort studies. Radicular pain regressed across the spectrum of our observations respectively in accordance to the above classification.

Discussion and Conclusion: Occurrence, diagnostic algorithms, clinical classification and surgical treatment of LEV must be assessed, identified and established to optimal algorithm for examining patients with LEV Combining Venospondylography with VAS as a screening method for verification of LEV, we optimised Clinical manifestations, diagnostic criteria and tactics.

We Optimised diagnostics tactics, patterns of frequency and eventually maximise surgical tactics in conjunction with adequate verification and established indications for surgical intervention in case of failure of complex conservative treatment for 6 months, or earlier with the progression of neurological symptoms in no less than 6 months. Conclusively, LEV should be considered in the differential diagnosis of spinal radiculopathy.
\end{abstract}

Keywords: Lumbar Varicosity; Lumbar Spine Varix; Venospondylograghy; Epidural Varices; Misdiagnosis 


\section{Abbreviations}

LEV: Lumbar Epidural Varix, VAS: Visual Analog Scale

\section{Introduction}

Back pain and radiculopathy are commonly caused by disc herniation or spinal stenosis. The prevalence of a lumbar epidural varicose vein lies between the rate of $0.067 \%-1.2 \%$ and is displayed across various studies [1-4]. Impingement of Lumbar epidural varices on nervous tissue has only rarely been described as an acquired pathology of the lumbar spine. This is manifested by the expansion of the internal vertebral venous plexuses [1]. However, this often accompanies the expansion of the external vertebral venous plexuses, intravertebral venous channels and the veins connecting them together [2-5].

Lumbar epidural varices (LEV) are characterized by the absence of arterial blood bypass, expansion of the epidural venous plexus, the increased pressure which follows the deposition of a large amount of blood. In the genesis of clinical manifestations, the pathology of the internal vertebral venous plexus (epidural veins) and intervertebral veins connecting the internal and external venous plexus is of primary importance. Enlarged veins located epidurally or in the intervertebral foramen cause compression or irritation of the dural sac and spinal roots [3-8]. The cardinal difference of varicose veins is the expansion of normal veins, without the formation of additional abnormal veins as typical venous malformations. LEV can develop as an independent process or accompany degenerative changes within the lumbar spine [2]. The diagnosis of epidural varicose veins is complex, hence often misinterpreted, and for the most part are diagnosed by chance during surgery [7]. Irrational and radical methods for occluding varicose veins compressing roots lead to significant venous bleeding. These factors determine the importance and the clinical significance of epidural varicose veins. In order to develop diagnostic criteria for $\mathrm{LEV}$, methods of surgical interventions, the immediate and long-term results of surgical treatment must be presented as illustrated in this study.

\section{Materials and Methods}

The following topographic and anatomical variants of varicose of epidural veins are hereby identified:
1. Segmental varicose veins (SVV), caused by the expansion of the intervertebral veins and as a rule, causing compression or irritation of the segmental roots passing along these veins through the intervertebral foramen;

2. Local varicose veins (LVV), due to the limited expansion of the ventral, less often dorsal longitudinal venous trunks, intervertebral veins at the level of 1 - 2 vertebrae, which usually causes compression of the dural sac, segmental roots;

3. Diffused or Widespread varicose veins (DVV) or (WVV) of the lumbar spine, associated with the extensive expansion of epidural veins, primarily the ventral epidural longitudinal venous trunks, less often the dorsal epidural longitudinal venous trunks. This type of varicose veins is usually combined with a significant increase in venous pressure throughout the epidural and, accordingly, vertebral venous system.

In this study, 100 patients with LEV were selected who had undergone microscopic decompression using micro-coagulation techniques were observed. Patients without operative verification of the diagnosis were excluded in this series. SVV was discovered in 65, LVV - in 27, and DVV - in 8 patients. All the patients were examined before surgery using MRI, venospondylography. During some of the observations, the modality applied and performed was electroneuromyography, all clinical symptoms were comprehensively evaluated. The results of each patient's surgical treatment was appropriately evaluated before the official discharge of patients and during follow up visits. During the initial postoperative period, all patients were subjected to an MRI scan, but not earlier than 6 months after the surgical intervention.

\section{Results}

Clinical symptoms: The main clinical sign of epidural varicose veins was the presence of radicular manifestations. Radicular pain syndrome was present in 60 patients with SVV, 25 - with LVV and 6 - with DVV. Sensory radicular abnormalities were observed in 50 patients with SVV, in 22 patients with LVV, and in 3 patient with DVV. Radicular motor impairment was observed in 58 patients with SVV symptoms, in 19 - with LVV, and in 5 - with DVV. Tension syndrome was not recorded or expressed as an abnormality or manifestation. Lumbalgia was observed in 44 patients with SVV, 24 patients - with LVV, and 8 patients - with DVV. 
As expected, patients with epidural varicose veins experienced significant fluctuations in neurological symptoms and most importantly, pain throughout the day. This was most typical for those with DVV followed by LVV. Often localized pains was not present in the right and left lower extremities, without clear somatotopic localization.

\section{Neuroimaging diagnosis of epidural varicose veins}

Normally, epidural veins on MRI were not visualized. The maximum severity of epidural varicosity during standard MRI examination was found in the form of "empty MR signal" zones, which repeated the contours of individual veins and main epidural venous trunks (ventral and extremely rarely dorsal venous trunks, intervertebral veins, venous conglomerates) (Figure 1). Venous epidural canals caused compression of the dural sac. The enlargement of intervertebral veins and compression of segmental roots caused by them were visualized (Figure 2). Partial thrombosis of varicose veins is rarely observed. In this case, a mixed MR signal was detected in the form of spotting with alternating sections of high and low signals in the T1 and T2 modes. In the presence of moderate blood flow throughout the varicose veins sometimes heterogeneous and amplified by contrast. During the T2 in a T1-weighted mode, the signal displayed was low, weighted mode, the signal varied depending on the level of blood flow - low if the blood flow was high, and high if the blood flow was low. As for Varicose vein thrombosis during the period of 5 days to 2 months, the MRI picture was due to the formation of methemoglobin and showed a high signal at T1 and T2 weighted modes. After 2 months hemosiderin dominated and the signal became low at T1 and T2 weighted modes. In the presence of partial thrombosis and recanalization of the vein, the introduction of contrast caused an increase in the signal along the periphery of the vessel.

In spinal selective angiography, the epidural venous plexus could not be contrasted. Two versions of the venospondylography technique have been developed; the introduction of contrast into the spinous process and the introduction into the vertebral body by puncture transdurally. Venospondylography was performed on an angiograph using subtraction to track the pathways of blood outflow.

After injection into the spinous process, contrast entered the posterior longitudinal and ventral longitudinal venous trunks connecting their veins, retrocorporeal veins and then into the inter-

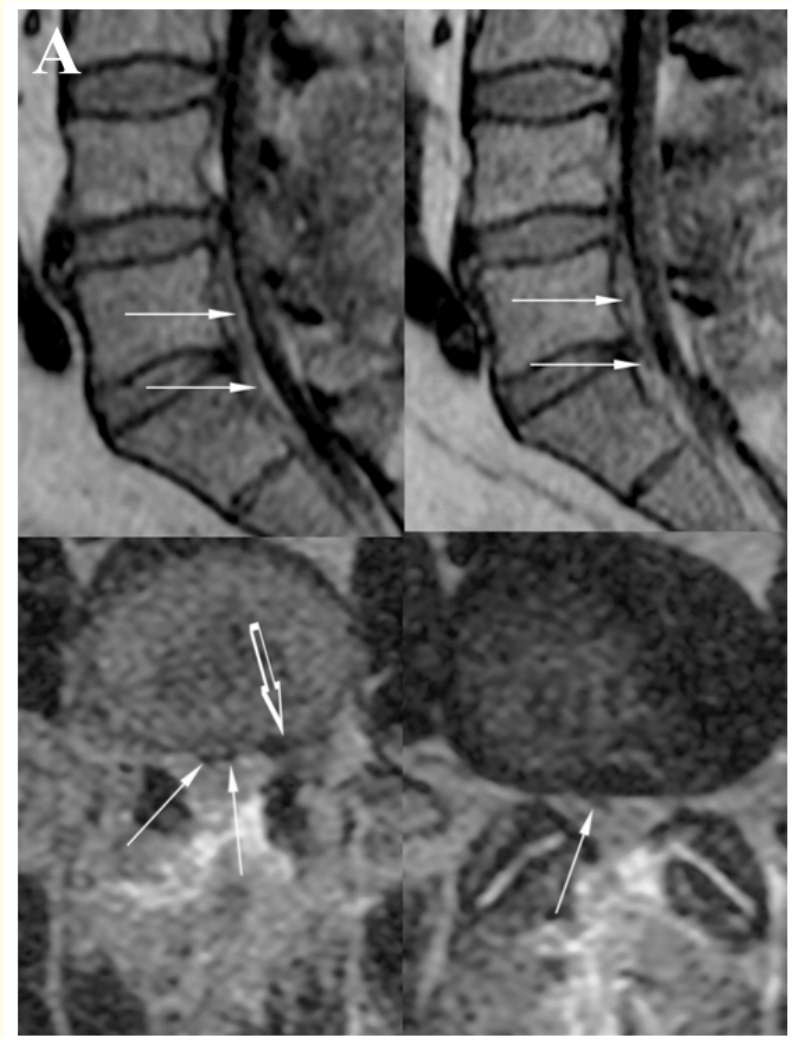

Figure 1: Segmental varicose veins of the epidural veins on MRI in T1-mode.

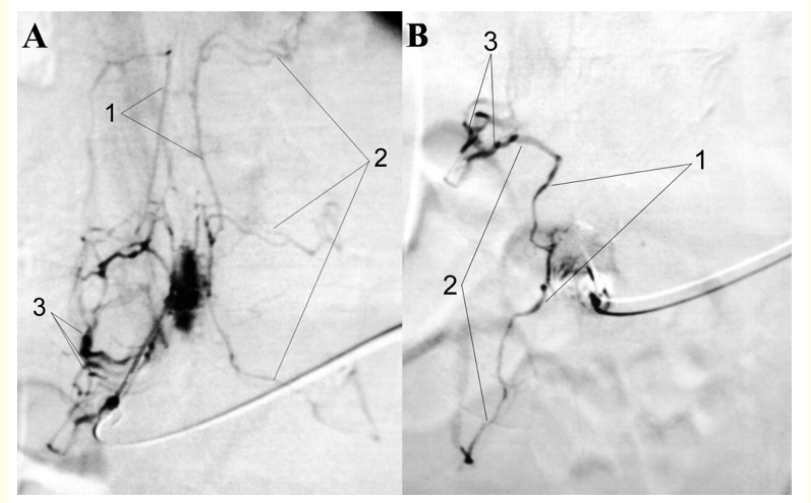

Figure 2: Segmental varicose veins of the epidural veins, dilatation of the intervertebral veins at the L5 - S1 level on the left. Bone subtraction venospondylography (A - into the spinous process of the body L5, B - into the body S1): 1 - ventral longitudinal venous trunks, 2 - normal segmental veins, 3 - varicose segmental veins L5 - S1 on the left. 
vertebral, basivertebral, ascending lumbar, segmental veins and the inferior vena cava (Figure $3 a$ and $3 b$ ).

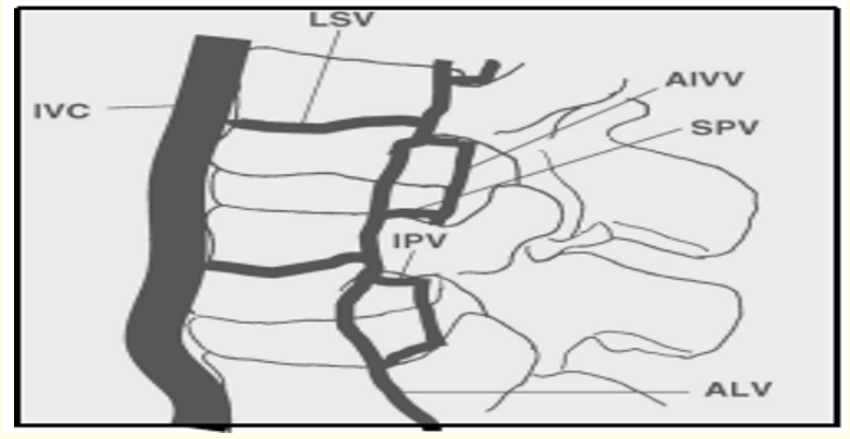

Figure 3: Diagrammatic representation of the lumbar veins of the spine (IVC: LSV: AIIV: SPV: IPV: ALV).

Simultaneously the contrast moved along the venous paths antegrade, along the blood stream. From a critical perspective, the application of contrast to the ventral longitudinal venous trunks, retrocorporeal veins, intervertebral veins is important, the vertebral body was first contrasted transdurally into the lumbar vertebral body. The display of outflow of the contrast from the body were twofold. Less often, contrast flowed through the basivertebral vein into the retrocorporeal veins, then the ventral longitudinal venous trunks and drained along them in the rostral direction. This pattern was observed with difficulty in blood flow in the inferior vena cava. Normal blood flow within the inferior vena cava, contrast, as a rule, flowed through the radial veins of the vertebral body into the external vertebral venous plexus, into the segmental veins, then the ascending lumbar veins or the inferior vena cava. This method was not always possible to contrast the epidural veins, which is why the transdural method of venospondylography in the vertebral bodies is of lesser importantance from a clinical point of view.

In the presence of epidural varicose veins, dilated and convoluted ventral longitudinal venous trunks, retrocorporeal veins, and intervertebral veins are contrasted. The expansion of the dorsal longitudinal venous trunks was extremely rarely. In the case of Segmental varicose veins (SVV), only the intervertebral veins experienced expansion at one or more adjacent levels, most often this is observed (Figure 4). Concerning local varicose veins (LVV), the expansion of the ventral longitudinal venous trunk more often on the one hand, connecting the ventral longitudinal venous trunks of the retrocorporeal veins and intervertebral veins at the level of 1 2 vertebrae. Finally concerning widespread varicose veins (WVV) of the lumbar spine, epidural veins widen over 3 - 5 vertebrae. The most pronounced was the expansion of both ventral longitudinal venous trunks, retrocorporeal and intervertebral veins. The greatest role in the clinical manifestations is played by the expansion of the ventral longitudinal venous tract and intervertebral veins.

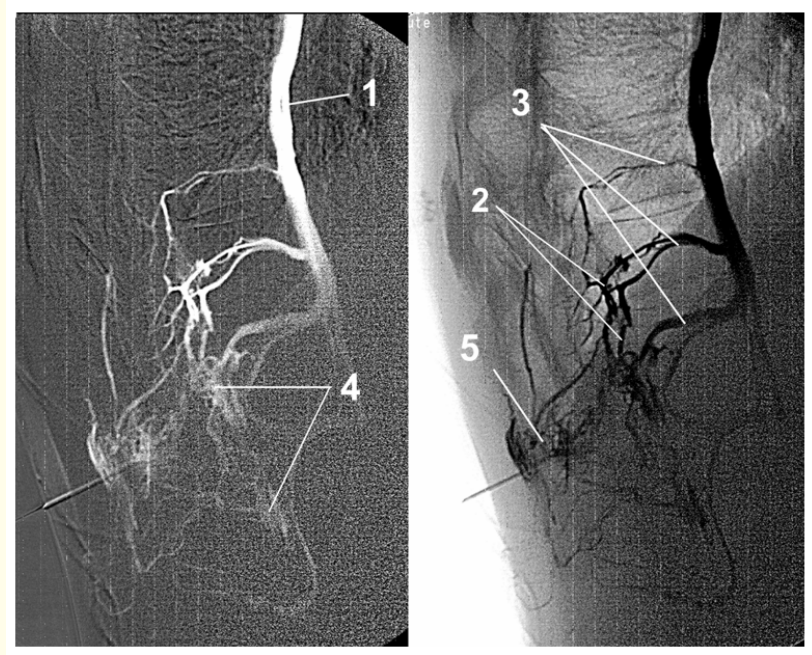

Figure 4: Local varicose veins of the epidural veins, expansion of the intervertebral veins and longitudinal venous trunks at the level of L1 - L2 - L3. Venospondylography, injection into the spinous process L2, lateral view:1 - ascending lumbar vein, 2 - varicose segmental veins L1 - L2 - L3, 3 - basivertebral veins, 4 - varicose longitudinal venous trunks, 5 - contrast in the spinous process.

Venous outflow from epidural varicose veins occurs in three ways. Firstly, the normal drainage pathway of the intervertebral veins, segmental veins flowing into the inferior vena cava, indicating patency of the inferior vena cava, low central venous pressure (CVP), normal pressure gradient (in the epidural veins, the pressure is higher than the pressure in the inferior vena cava). Secondly, through the ascending lumbar veins, indicating low CVP, but increased pressure in the caval system. Drainage bypasses the inferior vena cava, through the intervertebral and segmental veins that 
flow into the ascending lumbar veins, into the azygous and hemiazygous veins, and finally then into the superior vena cava (Figure 4). Finally, through the internal vertebral venous plexus (through the ventral longitudinal venous trunks) to the venous system of the thoracic spine - azygous and hemiazygous veins. In this case, the epidural veins appear circuitous for the outflow of blood from the inferior vena cava. Blood from the lower extremities enters the system of the inferior vena cava, due to the high pressure contained the flow continues into the epidural veins, and through them into the azygous and hemiazygous veins and eventually into the system of the superior vena cava. This drainage pathway is associated with high intra-abdominal pressure and difficulty in blood flow, both in the vena cava and in the ascending lumbar veins, or difficulty in blood flow in the inferior vena cava in combination with aplasia of the ascending lumbar veins. This type of drainage is accompanied by an unfavorable and progressive clinical course, often combined with varicose veins of the lower extremities.

\section{Surgical treatment of varicose veins of epidural veins}

The choice of surgical tactics depended on the type of varicose veins and clinical manifestations presented, the purpose of the chosen intervention was for decompression of the nerve structures. Additionally, the aim was to avoid the complete blockage of the blood flow in the epidural venous system, since many cases of varicose veins this was the only means of collateral blood flow (for example, caval hypertension). Surgical intervention selection was based on the analysis of venospondylography, MRI and clinical symptoms.

If unilateral radiculopathy presented as the dominant symptoms which was caused by the expansion of the intervertebral veins, and compression of the nerve roots (in the case of SVV), the surgical intervention aimed at coagulation and intersection of the intervertebral veins at the level of the affected roots. Coagulation and intersection of the ventral longitudinal venous trunk is indicated, if it causes irritation or compression of the dural root exit site (in case of local varicose veins). Varicose intervertebral veins coagulated and bisected no more than at the level of 2 vertebrae on one side as coagulation of a larger percentage of intervertebral veins could lead to significant impairment of blood flow (Figure 1).

In the presence of bilateral radicular symptoms caused by segmental or LVV, surgical intervention was aimed at bilateral coagu- lation and bisection of the intervertebral veins at the lesion level. Ventral epidural venous trunks coagulated and nipped only in the case of pronounced compression of the dural sac, and on the other hand (Figure 3 and 4).

Where epidural varicose veins were present due to increased pressure in the vena cava (all cases of DVV), the tactic of radical intervention with coagulating all varicose veins that compress the roots and dural sac quite often led to an increase in sensory and motor manifestations of radiculopathy. Therefore, an alternative intervention was applied - occlusion of varicose veins which is carried out in the dural zone of only those roots, where neurological symptomatology was displayed. The preservation of the ventral longitudinal venous trunks was focused on, as a result of this being a pathway of collateral blood flow (Figure 3).

The application of surgical interventions for LEV is a rather complex procedure as a technical term. SVV and damage to one root, as a rule, interlaminectomy and medial facetectomy are performed. SVV, LVV and a lesion of 2 - 3 roots, hemilaminectomy and medial facetectomy are performed at the site of the concerned roots. Subsequently, an operating microscope with an 8-10-fold increase. Preservation of root and lateral division of the dural sac to a sufficient extent. Large epidural veins are thin-walled, often located loosely among adipose tissue, less often fused with a periosteum, roots, or dural sac. Mobilization of the root without adequate visualization of the veins can lead to damage of the epidural veins and uncontrolled bleeding. Selection of epidural venous collectors from the root and lateral division of the dural sac is also performed under the control of an operating microscope. At the first stage, intervertebral veins around the root are selected and coagulated. When coagulating the intervertebral veins, care should be taken that the thermal effects of microcoagulation are not transmitted to the roots. To prevent rupture, the vein is coagulated by low-energy microcoagulation, gradually over the entire length several times (Figure 1-3). It shrinks with repeated, phased coagulation along the length of the vein. Within the center of the coagulated zone, the vein then bisects, next the lateral aspect of the dural sac, which is medially displaced, are mobilized. In this area, under the lateral aspects of the dural sac, there is a ventral longitudinal venous trunk. If its varicose veins and compression of the dural sac are detected, it is coagulated, 2 or 3 roots. The ventral longitudinal venous trunk coagulated only in the case of SVV or LVV, this coagulation is more complicated than intervertebral veins. The intervertebral veins are 
convoluted and not fixed, so coagulation does not lead to its tension. The ventral longitudinal venous trunk, as a rule, is not convoluted, sometimes fixed to the periosteum of the posterior surface of the vertebral bodies, as if "stretched" on the posterior surface of the body. The coagulation leads to a reduction in the trunk and an even greater tension, which does not allow adhesion of the walls of the veins, predisposed to rupture. If it is found that the venous trunk is fixed and stretched, it is necessary to coagulate it in a limited area, to achieve adhesion of the walls of the vein in this area. The trunk is then bisected and separated from the periosteum, causing weakens in the tension and after which the venous trunk is gradually coagulated over a larger extent.

Local varicose veins (LVV) caused by the expansion of the ventral longitudinal venous trunks, intervertebral veins at the level of 1 - 2 vertebrae and the absence of caval hypertension, the veins are occluded, the dural sac, and the roots are decompressed. In unilateral symptoms, interlaminectomy (one level of lesion) or hemilaminectomy ( 2 - 3 levels), medial facetectomy is performed. In bilateral symptoms, a bilateral interlaminectomy is performed (with a lesion at the same level), or a laminectomy (with a lesion at the level of 1 - 3 vertebrae). Intervertebral veins are coagulated and bisected around the concerned roots. The lateral aspects of the dural sac at the required level are mobilized and medially displaced, and the varicosed ventral longitudinal venous trunks coagulated (preferably only on one side). Retrocorporeal veins can also be coagulated using posterolateral approaches, their coagulation sometimes leads to separation of the basivertebral vein and severe bleeding from the vertebral body. In this case, the place of exit from the posterior surface of the body of the basivertebral vein is sealed with wax or a tightly twisted hemostatic sponge.

In the case of diffused Varicose Veins (DVV) of the lumbar spine and the expansion of all epidural veins, an increase in venous pressure was frequently observed throughout the epidural and accordingly, vertebral venous system. In such cases, coagulation and bisection are performed only in intervertebral veins in the area of clinically interested roots. The dural sac was then decompressed by performing a laminectomy, medial facetectomy. The ventral epidural longitudinal venous trunks was left intact where possible or coagulated on the one hand, given that this was often the only way of collateral blood flow (Figure 4). In 2014, Haydar Celik reported delayed-radiological recovery in LEV post surgical procedures and emphasized MRI follow-ups [3] as the surgically excised lesion become symptomatic again.

Treatment results: $n=100$, total varicose vein occlusion was performed in all 65 patients with segmental varicose veins, 27 patients with local varicose veins, total decompression was performed in 17 patients of the possible 27 patients. In patients with diffused types radical decompression was not performed, in all patients partial decompression of varicose veins was performed.

Observations showed a regression of neurological symptoms detected to some degree in all patients. Radicular pain syndrome regressed in 60 out of 65 patients with segmental varices symptoms, in 25 out of 27 - with LVV and 6 out of 8 - with extensive varices. Sensory radicular disturbances regressed in 50 out of 65 patients with segmental symptoms, in 22 out of 27 patients with LVV and 3 patients with DVV. Motor radicular disorders regressed in 58,19 and 5 respectively.

Long-term results indicated the total of 60, 22 and 5patients respectively showed improvement. Sometimes after the display of improvement, the disease progressed [3], however, the disease never led to gross neurological symptoms. Patients were always able to move independently; for a long time and their ability to work remained insignificantly altered.

\section{Discussion}

Spinal radiculopathy is a multifactorial disease with inflammatory, neuropathic, ischemic, and mechanical components [1,5]. Mechanical stress on the nerve root can impair intra-radicular blood flow and cause intra-neural damage and functional changes. Lumbar epidural varicose vein is a rare clinical condition that can lead to neurological deficits.

Three types of lumbar epidural varicose veins were described according to the magnetic resonance imaging (MRI) findings:

1. Type I - A thrombosed dilated epidural vein,

2. Type II - A non-thrombosed dilated epidural vein and

3. Type III - A sub-membraneous epidural hematoma [32]. 
The frequency of Lumbar epidural varices (LEV) is not precisely established despite several series that have been conducted $[7,8,10,11] .4 \cdot 5 \%$ of occurrence out of total 1091 operated patients with degenerative processes of the lumbar spine, the frequency was reported in a study while Paksoy Y and Gormus N., reported another $0.13 \%$ out of total observations of 9640 with lumbalgia and radicular symptoms, examined for a period of 2 years [12].

According to the pathomorphological manifestations of LEV, it was similar to varicose veins of the lower extremities [13]. The reported cases of epidural varicose veins are classified as SVV, LVV and DVV [10]. In the majority of cases described, the ventral longitudinal venous trunks, retrocorporeal veins, and intervertebral veins were dilated. Varicose dilated intervertebral veins were located near the axillary region of the root $[2,6,14]$.

Some authors believe that epidural varicose veins are similar in their pathogenesis to varicose veins of the lower extremities $[13,14]$. However, until now, the causes of lumbar epidural varicose veins have not been conclusively established. It was previously believed that large foramina or central hernias of the intervertebral discs led to severe compression of the epidural veins, impaired outflow of blood and resulted in epidural varicosity [11,15]. Simultaneously, a number of publications that discussed the presence of radicular symptoms caused by varicose veins, noted that it was not possible to detect clinically significant hernias of the intervertebral discs in the presence of stenosis at the intervertebral foramen. It is believed that a violation of the outflow of blood from the epidural venous plexus through the intervertebral veins into the vena cava system can lead to its expansion, engorgement and deposition of a large amount of blood [5,10]. At the same time, the outflow disturbance may be higher. In the study of Paksoy [12], patients with lumbalgia and radicular syndromes, a violation of blood flow in the inferior vena cava was found, which led to varicose veins of epidural and paravertebral veins. The age of patients ranged from 20 - 53 years, with the a average age being 30 years old. In the first group of 13 patients there was thrombosis of the inferior vena cava. The site of thrombosis was located below the inflow into the vena cava of the renal veins. In 2 patients, vena cava occlusion was present as a result of pregnancy. In the third group of 12 patients, the difficulty in blood flow was due to compression of the vena cava by the intraperitoneal volumetric process or mass. In all patients, the symptomatology debuted with acute radicular symptoms, to which lumbalgia joined [12]. Elimination (delivery in pregnant subjects and or treatment) of the cause of impaired blood flow in the vena cava led to a regression of neurological symptoms [12]. A case of varicose veins of the lumbar epidural veins as a consequence of thrombosis of the inferior vena cava by Campbell N. and colleagues [16]. Many cases of widespread or diffused varicose veins (DVV) of the lumbar epidural veins due to an increase in intra-abdominal pressure (obesity, pregnancy) or hypertension of unknown origin in the cava - venous system have also been described $[17,18]$.

Another possible reason for the development of varicose veins is pathological transient drainage of blood through the epidural venous network from the vena - cava system, which is facilitated by the absence of valves in the paravertebral veins, which leads to the formation of reverse blood flow [11,19] and suggest that hernias of the intervertebral discs cause compression, obstruction and or occlusion of the anterior part of the internal venous plexus (anterior longitudinal epidural venous trunks). Varicose veins dilatation is observed distally from the site of occlusion. Since there are no valves in the spinal veins, after occlusion of the venous blood flow from the internal venous plexus to the external, blood retrogradely flows from the external collectors into the internal venous plexus, leading to its expansion. Spinal veins are the collateral blood flow paths of the caval and unpaired (azygous) vein systems. However, in most cases, disc herniation is not associated with the occurrence of varicose veins of epidural veins $[1,10]$.

Zimmerman GA described the formation of epidural varicose veins with partial epidural vein thrombosis. He believe that an acute disc hernia led to epidural vein endothelial injury, and subsequently to their partial thrombosis [11]. Thrombosed veins can cause severe compression of the dural sac and spinal roots. Varicose expansion develops in veins distal to the site of thrombosis. On the basis of vertebral canal ultrasonography, it is believed that large disc hernias cause compression in addition to the nerve structures and the epidural venous plexus [20]. Chronic compression of these venous plexuses can lead to thrombosis of LVV. Jayson M. I. and colleagues, attempted to classify epidural varicose veins on the basis of radiological, anatomical and physiological data. In type I, there is a thrombosed vein (usually a ventral longitudinal venous trunk, sometimes a segmental vein). Usually, acutely -developed hernias of the lumbar discs [21]. Type II, varicose veins of segmental vein without thrombosis. The authors believe that this may be a 
post-thrombotic dilatation of a vein due to its recanalization. Type III, epidural hematoma due to bleeding from varicose veins [22].

In the work of Gundry CR., it's suggested that acutely developed herniated discs with ruptured posterior longitudinal ligaments can lead to rupture of varicose epidural veins, and hematoma [22,23]. This is possible in the presence of an epidural cicatricial process and fusion of veins with a posterior longitudinal ligament. From the above classifications (Type I-III), varicose veins of the epidural veins do not exist on their own, but only as post-thrombotic dilation of the veins due to their recanalization. Together, this cannot explain the cases of DVV of the entire lumbar region, since it is difficult to imagine that all these veins were thrombosed and then recanalized. In such a situation, the frequency of epidural vein thrombosis should sharply increase, which is manifested by an acute onset of the disease. However, with varicose veins, clinical symptoms develop gradually over the course of years and even decades.

There are studies trying to link varicose veins with a violation of the structure of the venous wall, while others observed changes in the structure of the venous wall associated with the expansion of the vein and secondary rearrangements of the venous wall due to the constant increased pressure contained within. There is currently no convincing data on the violation of the chemistry of collagen, elastin, and other components of the venous wall that would lead to a change in the resistance of the vein to intravascular pressure [2].

The clinical manifestation of epidural varix can be associated with two mechanisms:

1. Compression of the dural sac and roots by dilated veins or irrigation of roots by dilated veins;

2. Increased pressure in the epidural veins, transmitted to the perimedular veins that drain the spinal cord and roots. This leads to difficulty in the outflow of blood from the nervous structures, which is clinically manifested by slowly progressive myelopathy and radiculopathy [14,24]. Mostly, epidural varicosity manifested by radiculopathies, and extremely rarely by myelopathies $[11,17,25]$. Most authors believe that radiculopathies develop as a result of compression of the spinal cord or roots by the expanded epidural veins $[1,9]$.
Some authors suggested that myelopathy may also be due to impaired drainage of the vessels of the spinal cord due to hypertension in the epidural venous plexus [12]. However, in many studies the presence of myelopathy in lumbar epidural varicosity is disputed [2]. Quite constant with varicose veins is lumbalgia. However, its cause remains unknown [12].

In many cases, LEVs are found against the background of stenosis, spondylarthrosis, hernias of the intervertebral discs, in these cases, isolating the symptoms caused by varicose veins from other processes is difficult $[8,24]$.

For the diagnosis of LEV, both conventional MRI and non-standard MRI protocols are used. Thus, spin echo T1 weighted regimen with intravenous reinforcement by a magnet, according to some authors, it is the best non-invasive method for examining the epidural space $[26,27]$. The turbo-spin echo T2 method is a weighted mode with contrasting magnetic or STIR sequence (short T1 inversion recovery) giving inconsistent results due to insufficient spatial resolution. Nevertheless, the most informative and accurate diagnosis of LEVs is the use of venospondylography [28]. Venospondylography allows the establishment of the type of varicose veins, localisation and the place of neurovascular conflict [29,30]. Venospondylography was performed on an angiograph using subtraction, which allowed the path of blood outflow to be trace and permit the clear visualisation the veins. Genevay S., in 2002, considered that in all cases of varicose veins with neurological symptoms, decompression of nerve structures is necessary [24]. So Slin'ko E., performed decompression of nerve structures as well as discectomy with and without interfering in the veins [33]. Other authors end the operation with an intervention on the veins. Cases have been described when coagulation of veins was used [1,11-12,25,29,33] blood aspiration and dissection of varicose veins, [2,27] compression of varicose epidural veins with hemostatic sponge [6]. In most cases, the interventions resulted in a positive clinical outcome with regression of neurological symptoms [1,29].

With DVV, surgical interventions on the epidural veins are usually ineffective and do not significantly affect the course of the disease [1]. This is due to insufficient radicality of surgical interventions and inadequate surgical tactics in the case of DVV and caval hypertension. Much more effective interventions leading to the elimination of hypertension in the inferior vena cava [12]. Un-

Citation: Kayode Agboola and Eugene Slynko. "Diagnosis and Surgical Treatment of Epidural Varicose Veins of the Lumbar Spine". Acta Scientific Neurology 4.3 (2021): 03-12. 
fortunately, according to the data, it is not always possible to find the cause of hypertension in the inferior vena cava and to radically eliminate it.

\section{Conclusion}

Conclusively, epidural varicose veins is a specific nosological form of spinal pathology which despite the use of modern imaging methods (such as MRI, myelography and CT), yet can be often challenging to diagnose and distinguish from several other lumbar pains causes [5,29,31]. The diagnosis and surgical treatment are complex and are consistently being developed. Furthermore, with adequate diagnosis using careful history taking, clinical examinations, venospondylography and modern neuroimaging (high definitive MRI and MRI angiography, 'CT'), a justified surgical tactics may be implored with resultant positive outcomes. The observations presented, after surgical treatment, in most cases displayed and noted an improvement in the neurological condition of patients.

\section{Conflict of Interest}

Nil.

\section{Financial Support and Sponsor}

Nil.

\section{Bibliography}

1. Chin-Ho Wong., et al. "Symptomatic Spinal Epidural Varices Presenting With Nerve Impingement". SPINE 28.17 (2003): E347-E350.

2. Gul Moonis., et al. "Intradural venous varix: a rare cause of an intradural lumbar spine lesion 28.20 (2003): E430-E432

3. Haydar CELIK., et al. "Lumbar Epidural Varicose Vein: Early Neurological Improvement and Late Radiological Full Recovery with Surgery; The Importance of Magnetic Resonance Imaging in Follow-Up". Turk Neurosurg 25.5 (2015): 824-827.

4. Hee Joon Jeong., et al. "Severe lumbar radiculopathy with epidural venous plexus engorgement in a morbidly obese pediatric patient". Medicine 98 (2019): 33.

5. Stefan Endres. "Epidural varicosis as a possible cause of radicular pain: a case report". Journal of Medical Case Reports 5 (2011): 537.
6. Berthelot JM., et al. "Stretching of roots contributes to the pathophysiology of radiculopathies". Joint Bone Spine 85 (2018): 41-45.

7. Pekindil G and Yalniz E. "Symptomatic lumbar foraminal epidural varix". Case report and review of the literature". British Journal of Neurosurgery 11.2 (1997): 159-160.

8. LaBan MM., et al. "Varicosities of the paravertebral plexus of veins associated with nocturnal spinal pain as imaged by Magnetic resonance". American Journal of Physical Medicine and Rehabilitation 78.1 (1999): 72-76.

9. LaBan MM and Wang AM. "Engorgement of the cervical spinal epidural venous plexus simulating a spinal epidural mass". American Journal of Physical Medicine and Rehabilitation 93 (2014): 634-635.

10. Subbiah M and Yegumuthu K. "Lumbar epidural varices: an unusual cause of lumbar claudication". The Indian Journal of Orthopaedics 50 (2016): 440-443.

11. Kawai M., et al. "A case of idiopathic spinal epidural lipomatosis presented with radicular pain caused by compression with enlarged veins surrounding nerve roots". Acta Neurologica Scandinavica 105 (2002): 322-325.

12. Zimmerman GA., et al. "Symptomatic lumbar epidural varices. Report of two cases". Journal of Neurosurgery B 80 (1994): 914-918.

13. Paksoy Y and Gormus N. "Epidural venous plexus enlargements presenting with radiculopathy and back pain in patients with inferior vena cava obstruction or occlusion". Spine (Phila Pa 1976) 29 (2004): 2419-2424.

14. Demaerel P., et al. "Sciatica caused by a dilated epidural vein: MR findings". European Radiology 9 (1999): 113-114.

15. Hiroharu Kataoka., et al. "Venous Congestion Is a Major Cause of Neurological Deterioration in Spinal Arteriovenous Malformations". Neurosurgery 48.6 (2001): 1224-1230.

16. Hammer A., et al. "Localized venous plexi in the spine simulating prolapse of an intervertebral disc: A report of six cases". Spine 28 (2003): E5-E12.

17. Kim HJ., et al. "Obesity is independently associated with spinal anesthesia outcomes: a prospective observational study". PLoS One 10 (2015): e0124264. 
18. Campbell DN., et al. "Traumatic thrombosis of the inferior vena cava". Journal of Trauma 21 (1987): 413-415.

19. Shinichi Hida., et al. "Intraoperative Measurements of Nerve Root Blood Flow During Discectomy for Lumbar Disc Herniation". SPINE 28.1 (2003): 85-90.

20. Raghavendra V., et al. "Spinal Epidural Varices, a great Mimic of Intervertebral Disc Prolapse - A Case Series". Journal of Orthopaedic Case Reports 4.4 (2014): 3-5.

21. Dudeck O., et al. "Epidural venous enlargements presenting with intractable lower back pain and sciatica in a patient with absence of the infrarenal inferior vena cava and bilateral deep venous thrombosis". Spine 32 (2007): E688-E691.

22. Jayson MI. "The role of vascular damage and fibrosis in the pathogenesis of nerve root damage". Clinical Orthopaedics 279 (1992): 40-48.

23. Hiroyoshi Akutsu., et al. "A case of nontraumatic spinal epidural hematoma caused by extradural varix: consideration of etiology". The Spine Journal 3 (2003): 534-538.

24. Cooper R., et al. "Epidural Hematoma of the Lumbar Spine: 18 Surgically Confirmed Cases”. Radiology 187 (1993): 427-431.

25. Genevay S., et al. "Lumboraticulopathy due to epidural varices: two case reports and review of the literature". Joint Bone Spine 69 (2002): 214-217.

26. Hanley EN., et al. "Lumbar epidural varix as a cause of radiculopathy". Spine 19 (1994): 2122-2126.

27. Cohen I. "Extradural varix simulating herniated nucleus proposes". Journal of the Mount Sinai Hospital 8 (1941): 136-138.

28. Demaerel P., et al. "Sciatica caused by a dilated epidural vein: MR findings". European Radiology 9 (1999): 113-114.

29. Slin'ko EI and Al-Qashqish II. "Surgical treatment of lumbar". Journal of Neurosurgery Spine 5 (2006): 414-423.

30. Yucesoy K., et al. "Acute foot drop caused by thrombosed epidural vein". Acta Neurochirurgica 143 (2001): 631.

31. Zozulya YuA., et al. "Surgical Treatment for Pain due to Lumbosacral Nerve Root Compression". Kiev (2006): 348.

\section{Assets from publication with us}

- Prompt Acknowledgement after receiving the article

- Thorough Double blinded peer review

- Rapid Publication

- Issue of Publication Certificate

- High visibility of your Published work

Website: www.actascientific.com/

Submit Article: www.actascientific.com/submission.php

Email us: editor@actascientific.com

Contact us: +919182824667 